\title{
TRICHOPHYTON GLABRUM SABOURAUD 1910 PEUT-IL ÊTRE CONSIDÉRÉ COMME UNE VARIETÉ FIXE DE T. VIOLACEUM BODIN 1902 ?
}

Par J. BIGUeT, G. CocheT, M. DOBY-DUBoIS, S. MULLET et S. DEBLOCK

En 1910, Sabouraud considérait Trichophyton glabrum comme une variété fixe, satellite de $T$. violaceum (1), dont il ne différait que par une culture plus vivace et des colonies dépourvues de pigment violet et centrées par des papilles épaisses et grasses ; cette description de $T$. glabrum ne reposait à l'époque que sur l'étude d'une souche unique. Dès 1902, Bodin avait précisé que $T$. violaceum se colorait en violet aubergine en trois semaines environ et que la pigmentation se limitait parfois à un secteur des colonies ou même seulement à l'acumination centrale. Sabouraud ajoutait, en 1910, que la teinte violette tendait à disparaìtre après un ou plusieurs repiquages.

Catanei aussi estimait bien-fondée la distinction spécifique de $T$. violaceum. Parmi les travaux de cet auteur, qui avait eu l'occasion d'étudier de multiples souches de Trichophyton d'origine algérienne, nous retiendrons les faits suivants, publiés par lui, en 1931 et 1933 :

$1^{\circ} T$. glabrum est d'une culture plus lente que $T$. violaceum, contrairement à ce qu'écrivait Sabouraud en 1910. (Mais nous avons dit qu'il n'avait disposé que d'une seule souche).

$2^{\circ}$ Les colonies de $T$. violaceum se reconnaissent même en l'absence de pigment, dès la fin de la première semaine (cultures à $28^{\circ}$ ), parce qu'elles sont assez régulièrement arrondies, alors qu'au début les colonies de $T$. glabrum sont plus petites, grêles et irrégulières.

$3^{\circ}$ La pigmentation des souches algériennes apparait dès la première ou la deuxième semaine, et elle est généralement parfaitement développée la troisième semaine.

(1) T. violaceum, isolé par Sabouraud en 1892, fut décrit par cet auteur la même année, puis en 1895. Mais le champignon ne fut nommé que par Bodin en 1902. Il est curieux de constater que Sabouraud, en 1910, omette de citer Bodin dans son livre « Les teignes » lorsqu'il fait l'historique de l'espèce. Précisons que Bodin avait rendu hommage à Sabouraud en écrivant $T$. violaceum Sabouraud.

Ann. de Parasitologie, T. XXXI, $\mathrm{N}^{\circ} 4 .-1956$. 
$4^{\circ}$ L'inoculation à un Macacus inuus de cultures d'un $T$. violaceum ne sécrétant plus de pigment a permis des rétrocultures à pigmentation violette normale (1).

Enfin, en 1950, Vanbreuseghem acceptait aussi, mais avec des réserves, l'individualité des deux espèces. Il écrivait : « Jusqu'à présent, nous admettons avec Catanei que le $T$. violaceum et le T. glabrum sont deux espèces différentes, dont l'un, le premier, forme un pigment violet qu'il peut perdre, tandis que l'autre, T. glabrum, n'en forme pas. Catanei voit entre la forme non pigmentée du $T$. violaceum et le $T$. glabrum de petites différences morphologiques et d'autres dans la rapidité du développement des colonies. Elles ne nous sont pas apparues aussi nettement que Catanei le dit. »

Cependant, en Amérique, Emmons (1934), puis Conant (et ses coll., 1943 et 1954), faisaient tomber $T$. glabrum en synonymie devant $T$. violaceum ; cet avis était partagé en France par Langeron qui précisait, en 1945, que de nombreux ensemencements lui avaient fourni tous les intermédiaires entre le violet foncé et le blanc pur, et qu'il se trouvait en présence d'un phénomène identique à celui présenté par $T$. rubrum ou $T$. rosaceum. Toutefois, d'après Vanbreuseghem (1950), Langeron, alors qu'il préparait la deuxième édition de son «Précis de mycologie», avait écrit une révision de la classification établie jadis en 1930 avec Milochevitch. Dans cette classification modifiée, T. glabrum était individualisé en tant que sous-espèce de $T$. violaceum, aux côtés de $T$. gourvili (Catanei, 1932) et $T$. marginatum (Muys, 1921).

Etant donné ces opinions divergentes, la parenté de $T$. violaceum et de $T$. glabrum ne faisant d'ailleurs aucun doute pour personne, nous avons désiré, à la lumière de multiples ensemencements, reconsidérer la position précise de $T$. glabrum vis-à-vis de $T$. violaceum : ou bien identité absolue, ou bien variété très voisine, mais distincte et fixe.

(1) En 1938, Catanei a étudié sur le cobaye le pouvoir pathogène de $T$. violaceum et $T$. glabrum cultivés sur gélose à la farine de riz, qui lui ont permis d'obtenir des appareils conidiens parfois nombreux. Dans une première série d'expériences (culture broyée dans la vaseline stérilisée et appliquée sur la peau scarifiée) deux souches violettes sur six se sont révélées pathogènes et aucune des cinq souches de $T$. glabrum; dans une deuxième série d'expériences (inoculation réalisée sans vaseline), deux souches sur trois de $T$. violaceum et une souche de $T$. glabrum ont provoqué une lésion teigneuse. 


\section{Résumé de nos observations personnelles}

Nous avons disposé de 1.057 souches de Trichophyton à cultures glabres provenant de prélèvements effectués en Tunisie en 1950 et 1953 .

Parmi les souches recueillies en 1950, le comportement de 55 d'entre elles étiquetées primitivement T. glabrum a d'abord attiré notre attention; c'est pourquoi nous avons par la suite réalisé une étude particulièrement soigneuse d'un groupe de 382 ensemencements de la récolte de 1953.

Nous exposerons d'abord nos observations concernant la pigmentation de ces cultures, effectuées à $28^{\circ}$ (comme le préconise Catanei, 1933), sur milieu glucosé à $2 \%$ de Sabouraud :

$1^{\circ} 251$ souches sur 382 développèrent la coloration violet aubergine typique en moins de quatre semaines après l'ensemencement ; 7 furent violettes dans la deuxième semaine, 54 dans la troisième, les autres dans la quatrième. Ainsi, les souches tunisiennes de $T$. violaceum les plus typiques développent leur pigment plus lentement que les souches algériennes de Catanei.

$2^{\circ} 13$ autres souches se sont colorées dans un délai d'un mois, mais leur teinte était rose, et 6 ont montré tardivement, entre sept semaines et trois mois, des points ou des taches violets.

$3^{\circ} 75$ souches se pigmentèrent au cours du deuxième mois seulement; 20 devinrent d'un violet franc (4 incomplètement); 14 restèrent d'un violet clair et 41 roses. Sur 9 de ces dernières devaient apparaître entre la septième et la dixième semaine des points ou des taches violets.

$4^{\circ}$ Nous considérons maintenant 43 cultures de ce lot de 312 souches, en même temps que les 55 cultures d'un autre lot antérieurement dénommées $T$. glabrum.

Il s'agit, en effet, de 98 souches au total dont les colonies, après deux mois de culture, ne présentaient aucune coloration rose, mauve ou violette, même partielle, et demeuraient blanches ou jaunâtres.

a) Sur 19 de ces souches apparurent tardivement des points violets; 5 le troisième mois, 7 le quatrième, 4 le cinquième, 2 le sixième, la dernière enfin le neuvième mois.

b) Les 79 souches restantes se desséchèrent sans se pigmenter, mais 12 d'entre elles donnèrent lieu à des subcultures qui développèrent du pigment violet, 8 dans le délai habituel d'un mois, 3 durant le deuxième mois, une seule dans le courant du troisième mois de culture. 
Quelques cultures tertiaires seulement furent réalisées à partir de 67 subcultures restées blanches; l'une d'elles se pigmenta en un mois.

Nous compléterons ces observations par quelques remarques concernant la croissance des cultures et leur aspect macroscopique en fonction de l'apparition ou du défaut d'apparition du pigment :

$1^{\circ}$ Les cultures primaires à évolution rapide (c'est-à-dire se manifestant approximativement dans les 5 à 6 premiers jours qui suivent l'ensemencement et ayant par la suite un développement également rapide) ont fourni des colonies tantôt violettes (après un début jaune), tantôt blanches (1) ; les cultures pigmentées étaient habituellement bombées et presque sphériques, celles qui demeuraient blanches étaient moins denses au début, très étalées sur un grand diamètre. Ces cultures violettes et blanches, très vivaces, à surface humide et luisante, ont un mycélium qui plonge profondément dans la gélose ; elles correspondent au type I des cultures faviformes décrit par Sabouraud en 1929.

Les cultures primaires à évolution lente ont généralement donné naissance à des colonies d'abord blanches, puis se colorant en rose. Leur aspect général est assez semblable à celui des colonies violettes, mais elles sont souvent plus petites, plus coniques; elles semblent pouvoir se détacher facilement du milieu gélosé et deviennent vite sèches et rugueuses; elles évoquent le type II des cultures faviformes de Sabouraud.

Nos recherches diffèrent par conséquent de Catanei (1933), qui avait noté la correspondance des souches algériennes à cultures lentes avec la variété blanche, $T$. glabrum.

$2^{\circ}$ Les remarques précédentes ne sont valables que pour la majorité des cultures blanches, roses ou violettes. Nous avons observé, en effet, des souches qui s'étalent largement et prennent l'aspect des souches destinées à rester blanches. D'autre part, quelques souches blanches (5 sur un lot de 382 ensemencements) ont poussé lentement et ont présenté l'aspect macroscopique des colonies roses.

$3^{\circ}$ Il est possible que l'aspect macroscopique des cultures primaires dépende en partie du temps écoulé entre le prélèvement et la date de l'ensemencement (2). C'est ainsi qu'en ce qui concerne les

(1) Nous parlons des souches qui sont toujours demeurées blanches au cours des cultures primaires et des subcultures.

(2) Catanei, repiquant des cheveux teigneux conservés au laboratoire depuis un temps variant de quelques mois à quelques années, signale, en 1936, avoir obtenu une culture atypique d'un Trichophyton violaceum (cheveux conservés 
cultures blanches ou roses du lot de 382 ensemencements, les colonies à pousse lente du type I de Sabouraud furent presque toutes obtenues par l'ensemencement, effectué en mars 1954, de prélèvements vieux de cinq mois, tandis que les colonies vivaces, bien humides et luisantes, provenaient généralement de l'ensemencement en janvier de prélèvements qui ne dataient que de trois mois.

Rappelons à ce sujet que Joyeux (1914) a observé à propos de son Trichophyton soudanense des variations culturales qu'il estimait liées à l'époque de l'année où s'effectuaient les cultures.

\section{Conclusion}

Nos observations ont particulièrement porté sur 437 souches de Trichophyton glabres, d'origine tunisienne. Parmi elles, 96 auraient pu être nommées $T$. glabrum, puisque, à la fin du deuxième mois de culture, elles étaient encore dépourvues de toute pigmentation. En fait, l'examen prolongé de ces cultures durant près d'un an, celui de leurs subcultures secondaires et de quelques subcultures tertiaires ont montré que le pigment pouvait se développer partiellement ou complètement de façon extrêmement tardive. Comme, par ailleurs, aucune autre distinction morphologique n'a pu être établie de façon constante entre les souches violettes et celles qui sont demeurées blanches, nous sommes amenés à conclure que T. glabrum n'a aucune individualité réelle, même en tant que variété, et que les cultures blanches des Trichophyton glabres ne représentent que l'un des aspects morphologiques possibles des cultures de $T$. violaceum.

\section{BIBLIOGRAPHIE}

Bodin (E.). - 1902. Les champignons parasites de l'homme, Edit. Masson et $\mathrm{C}^{10}$, Gauthier-Villars, Paris.

Catiner (A.). - 1931. Remarques sur la valeur de la distinction spécifique des Trichophyton violaceum et glabrum. C.R. des Séances de la Soc. de Biol., CVI, p. 80.

- 1933. Etude sur les teignes. Arch. Inst. Pasteur d'Algérie, XI, p. 267.

16 mois). Sur cinq séries de repiquages, l'une seulement présenta tardivement des îlots violacés qui s'étendirent à la longue. Les subcultures à partir de fragments, pigmentés ou non, donnèrent soit des colonies tachetées, soit des colonies grisâtres, teinte prise fréquemment par Trichophyton violaceum conservé depuis longtemps dans les collections. 
1936. Caractères des cultures de champignons des teignes provenant de cheveux parasités depuis longtemps. C.R. des Séances de la Soc. de Biol., CXXIII, p. 1124.

1938. Sur les rapports entre les caractères des cultures des Trichophyton violaceum et glabrum, et leur pouvoir pathogène pour les animaux. Ibid., CXXVIII, p. 255.

Conant (N. F.), Martin (O. S.), Smith (D. T.), Baker (R. D.) et Callaway (J. L.). - 1954. Manual of clinical Mycology, Edit. Saunders Company, Philadelphie et Londres, $1^{\text {re }}$ édition $1945,2^{\circ}$ édition.

Emsons (C. W.). - 1934. Dermatophytes. Natural grouping based of the form of the spores and accessory organs. Archiv. of Dermat. a. Syphil., XXX, p. 337 .

Joyeux (C.). - 1914. Contribution à l'étude des teignes africaines, Trichophyton soudanense, Archiv. de Parasitologie, XVI, p. 449.

Langeron (M.). - 1945. Précis de Mycologie, Masson édit., Paris.

- et Mrlochevitch (S.). - 1930. Morphologie des Dermatophytes sur milieux naturels et milieux à base de polysaccharides. Essai de classification (deuxième mémoire). Ann. Para. Hum. et Comp., VIII, p. 465.

Sabouraud (R.). - 1910. Contribution à l'étude de la Trichophytie humaine. Ann. de Dermat. et de Syph., 30 novembre 1892, in Sabouraud.

- 1893-1894. Lcs Trichophyties humaines, Thèse Doct. en Médecine, Paris.

- 1910. Les teignes, Masson et $\mathrm{C}^{\text {io }}$ édit., Paris.

- 1929. Généralités concernant les Dermatophytes, $2^{\circ}$ mémoire. Le problème des cultures glabres. Ann. de Derm. et de Syph., X, p. 345.

Vanbreuseghem (R.). - 1950. Diagnostic et systématique des Dermatophytes. Contribution à la connaissance des teignes du Congo Belge. Ann. de la Soc. Relge de Méd. Trop., XXX, p. 865.

(Travail du Laboratoire de Parasitologie de la Faculté de Médecine de Lille. - Directeur : Professeur F. Coutelen). 